\title{
Tuberculosis Treatment Outcome among HIV Co-infected Patients at Mizan- Aman General Hospital, Southwest Ethiopia: A Retrospective Study
}

Fiseha $\mathrm{T}^{1 *}$, Gebru $\mathrm{T}^{1,2}$, Gutema $\mathrm{H}^{2,3}$ and Debela $\mathrm{Y}^{2,3}$

${ }^{1}$ Department of Clinical Laboratory Science, College of Medicine and Health Science, Wollo University, Dessie, Ethiopia

${ }^{2}$ Departments of Epidemiology, College of Health Science, Welkite University, Welkite, Ethiopia

${ }^{3}$ Department of Health Education and Promotion, School of Public Health, Bahir Dar University, Bahir Dar, Ethiopia

\begin{abstract}
Background: HIV-tuberculosis (TB) co-infection has emerged as a major public health threat throughout the world. According to the 2013 WHO global TB report, there were 1.1 million (13\%) TB/HIV co-infection, among these, the African Region (essentially sub-Saharan Africa) had by far highest TB/HIV co-infection (75\%). In 2012, WHO estimated 230,000 new cases of TB in Ethiopia (247 per 100,000); of whom 9819 (10\%) were HIV positive. On the other hand treatment outcome for HIV/TB co-infected patients are worse than those TB only patients. The aim of this study was to assess tuberculosis treatment outcome among HIV infected TB patients in southwest Ethiopia.
\end{abstract}

Methods: We analyzed the records of 2150 tuberculosis patients registered at Mizan Aman General Hospital from June 2010 to July 2013. Tuberculosis type and treatment outcome were categorized according to the national tuberculosis control program guideline. Data analysis was performed using the statistical package SPSS for windows, version 20.

Results: Of 2150 records reviewed, 397 (18.5\%) TB patients (210 male and 187 female) were found to be positive for HIV. Treatment success rates of HIV infected TB patients after completion of dose regimen was $29 \%$ (115). Of which, $4 \%$ (16) were cured, $24.9 \%$ (99) treatment completed, $1.3 \%$ (5) defaulted, and $5.5 \%$ (22) were died.

Conclusion: A high prevalence of HIV infection, and poor treatment success rate was observed among tuberculosis patients. A high proportion of TB patients' died, which is a serious public health concern that needs to be addressed urgently. Thus, to improve TB treatment outcomes TB/HIV integration as well as improved counseling and health education to reduce treatment interruption is required in our setting.

Keywords: Tuberculosis; HIV; Treatment outcomes

\section{Introduction}

Tuberculosis (TB) and HIV are a major public health challenges, particularly in resource-limited settings where there are minimal resources for infection control, diagnosis, and treatment [1]. In 2012, there were a total of 8.6 million incident TB cases (122 cases per 100,000 population) and 1.3 million death from the disease [2]. According to WHO global TB report (2013), there were 1.1 million (13\%) TB/ HIV co-infection. Among these, the African Region (essentially subSaharan Africa) had by far the highest TB/HIV co-infection (75\%). In 2012, WHO estimated 230,000 new cases of TB in Ethiopia (247 per $100,000)$; of whom 9819 (10\%) were HIV positive. Ethiopia ranks $7^{\text {th }}$ on the list of 22 countries that shoulder the highest TB burden.

The interaction between HIV and TB in persons co-infected with HIV and TB is bidirectional and synergistic. TB is the leading cause of morbidity and mortality in HIV-infected patients [3,4]. Similarly, infection with HIV is the most powerful known risk factor predisposing for Mycobacterium tuberculosis (MTB) infection and progression to active disease, which increases the risk of latent $\mathrm{TB}$ reactivation by 20-folds [5]. In addition to increasing individual susceptibility to TB following MTB infection, the increased burden of HIV-associated TB cases also increases MTB transmission rates at the community level [6].

In many countries, poor treatment and acquired HIV infection have been found to be the major reasons for the renaissance of TB [7]. TB treatment outcomes for HIV positive TB patients are worse than those for HIV-negative and HIV co-infection was associated with poor TB treatment outcomes (default and death) [8,9]. HIV-associated TB is related to an increased risk of TB deaths [10] and if HIV prevalence in the community continues to increase, however, it further affect the TB control program, by decreasing cure rates and increasing mortality and recurrent TB. Moreover, failure to complete TB treatment is associated with very high rates of recurrence especially in HIV-infected patients [11].

As the impact of HIV on TB, and the implications for TB and HIV control, has been acknowledged as a public health challenge in Ethiopia; understanding TB treatment outcomes of HIV infected TB cases may provide an indication of the effectiveness of national $\mathrm{TB}$ programmes. We, therefore conducted a retrospective study to explore TB treatment outcomes of HIV infected TB patients registered in Mizan Aman General Hospital, southwest Ethiopia. Treatment outcomes for coinfected patients are compared with those for TB patients in the province. Despite having a relatively well functioning national program in place, there are few published reports on HIV-TB co-infections from India.

*Corresponding author: Fiseha T, Department of Clinical Laboratory Science, College of Medicine and Health Science, Wollo University, Dessie, Ethiopia, E-mail: temafiseha@gmail.com

Received: November 20, 2014; Accepted: Juanuary 08, 2015; Published: January 20, 2015

Citation: Fiseha T, Gebru T, Gutema H, Debela Y (2015) Tuberculosis Treatment Outcome among HIV Co-infected Patients at Mizan-Aman General Hospital, Southwest Ethiopia: A Retrospective Study. J Bioengineer \& Biomedical Sci 5: 139. doi:10.4172/2155- 9538.1000139

Copyright: $\odot 2015$ Fiseha T, et al. This is an open-access article distributed under the terms of the Creative Commons Attribution License, which permits unrestricted use, distribution, and reproduction in any medium, provided the original author and source are credited. 
Citation: Fiseha T, Gebru T, Gutema H, Debela Y (2015) Tuberculosis Treatment Outcome among HIV Co-infected Patients at Mizan-Aman General Hospital, Southwest Ethiopia: A Retrospective Study. J Bioengineer \& Biomedical Sci 5: 139. doi:10.4172/2155- 9538.1000139

\section{Materials and Methods}

\section{Study setting}

This study was conducted in the southern region of Ethiopia in Mizan Aman General Hospital, Bench Maji Zone. Bench Maji Zone consistently has one of the highest HIV prevalence in southern region of Ethiopia. The hospitals was located in urban area and served as referral centers for health centers, community clinics and other private health facilities within the zone and two surrounding zones. The hospital has a TB clinic where patients with TB are registered and treated according the Directly Observed Therapy; Short-Course (DOTS) strategy operating under the National Tuberculosis and Leprosy Control Program (NTLCP) of Ethiopia [12]. The DOTS intervention includes providing recommended drug therapy for all registered $\mathrm{TB}$ cases, provider-initiated counseling and testing (PICT) of all registered TB cases for HIV, and ART linkage for antiretroviral therapy (ART) for all eligible HIV-positive TB cases.

\section{Study design and data collection}

A retrospective review of medical records of all tuberculosis patients registered from June 2010 to July 2013 at TB Clinic of Mizan Aman General Hospital for an estimation of prevalence of HIV and tuberculosis treatment outcome among patients with TB/HIV co-infection. The registration documents reviewed contain basic information such as patient's age, sex, disease status, tuberculosis type, HIV status, and treatment outcome.

\section{Statistical analysis}

Data were entered and cleared using Epidata version 3.1 and analysis was performed using the statistical package SPSS for windows, version 20. A summary descriptive analysis was made calculating various summary statistics; central tendency, percentages, and frequency was done.

\section{Ethical considerations}

The study was approved by Health Research Ethical Review Committee of Aman health Science College. Letter of co-operation was obtained from Bench Maji zone health department.

\section{Definitions}

Types of TB and treatment outcomes were defined according to the standard definitions of the NLCP adopted from WHO [13]. Types of TB was classified as: SPPTB (patient with one or more initial sputum smear examinations, direct smear microscopy, AFB-positive; or one sputum examination AFB positive plus radiographic abnormalities consistent with active pulmonary TB as determined by a clinician), smear-negative pulmonary TB (SNPTB: patient with pulmonary TB who does not meet the criteria for SPPTB), and Extra-pulmonary TB (EPTB: patient with TB of organs other than the lungs. Patients' treatment outcomes were classified as: cured (finished treatment with negative bacteriology result at the end of the treatment), treatment completed (finished treatment but without bacteriology result at the end of their treatment), defaulted (patients who interrupted their treatment for two consecutive months or more after registration), treatment failure (remaining smear-positive at five months despite correct intake of medication), died (patients who died from any cause during the course of treatment), transferred out (patients whose treatment result is unknown due to transfer to another health facility), and successfully treated (a patient who was cured or completed treatment).

\section{Results}

A total of 2150 TB patients reported in the TB clinic of Mizan Aman general hospital between July 2010 and June 2013. Of them, 397 were reported TB and HIV co-infection, which indicates $18.5 \%$ prevalence in this sample. Of whom, 210 (52.9\%), were males. The majority, 181 (46.6\%), of the HIV infected TB patients belonged to the age group of 15-29 years. Three hundred eighty six (97.2\%) of the HIV infected TB patients were new cases and the rest, $9(2.3 \%)$ were relapse cases and $2(0.5 \%)$ were return after defaulting. Out of the HIV infected TB patients, 261 (65.7\%) had SNPTB, followed by $70(17.6 \%)$ and 66 (16.7\%) who had SPPTB and EPTB, respectively (Table 1). Looking into the distribution of types of TB among HIV infected TB patients; the proportion of SNPTB, SPPTB and EXPTB was $65.7 \%, 19.5 \%$ and $14.8 \%$ among males respectively (Table 2 ).

The overall treatment outcome of HIV infected TB patients were 16 (4.0\%) cured, 99 (24.9\%) completed the treatment, 5 (1.3\%) defaulted, 22 (5.5\%) died, and $225(64.2 \%)$ were transferred out. No treatment failure was recorded in HIV-positive TB patients. The Treatment Success Rate (TSR) of all types of TB among HIV infected TB patients was 115 (29.0\%). TSR among HIV infected SPPTB patients was 23 (32.9\%), and was $69(26.4 \%)$ and 23 (34.8\%) among HIV infected SNPTB and EXPTB patients, respectively (Table 3).

\section{Discussion}

HIV co-infection among TB patients is well recognized as a major

\begin{tabular}{|c|c|c|}
\hline Characteristics & Frequency & Percent \\
\hline \multicolumn{3}{|l|}{ Sex } \\
\hline Male & 210 & 52.9 \\
\hline Female & 187 & 47.1 \\
\hline \multicolumn{3}{|l|}{ Age group } \\
\hline $0-14$ years & 22 & 5.6 \\
\hline $15-29$ years & 186 & 46.6 \\
\hline $30-44$ years & 153 & 38.5 \\
\hline $45-59$ years & 31 & 7.8 \\
\hline$\geq 60$ years & 6 & 1.5 \\
\hline \multicolumn{3}{|l|}{ Disease status } \\
\hline New & 386 & 97.2 \\
\hline Relapse & 9 & 2.3 \\
\hline Return after default & 2 & 0.5 \\
\hline \multicolumn{3}{|l|}{ Types of TB } \\
\hline SPPTB & 70 & 17.6 \\
\hline SNPTB & 261 & 65.7 \\
\hline EXPTB & 66 & 16.6 \\
\hline
\end{tabular}

Table 1: Socio-demographic and clinical characteristics of HIV infected TB patients $(\mathrm{N}=397)$ in Mizan Aman General Hospital, 2010-2013.

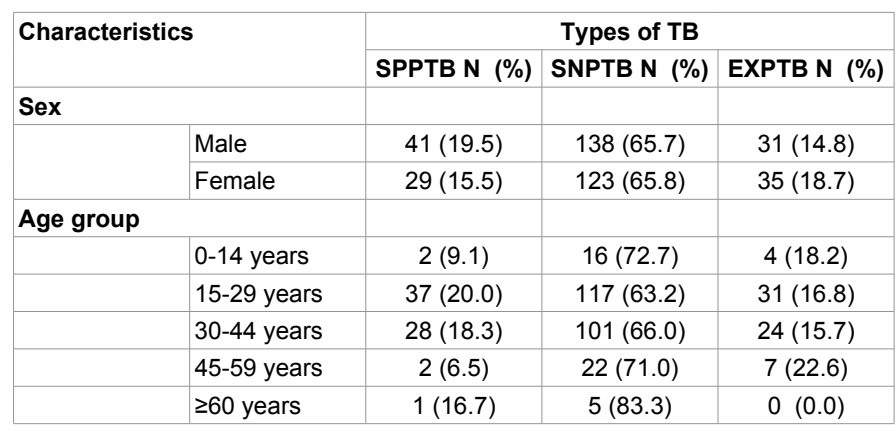

Table 2: Distribution of types of TB among HIV infected TB patients by sex and age in Mizan Aman General Hospital, 2010-2013. 
Citation: Fiseha T, Gebru T, Gutema H, Debela Y (2015) Tuberculosis Treatment Outcome among HIV Co-infected Patients at Mizan-Aman General Hospital, Southwest Ethiopia: A Retrospective Study. J Bioengineer \& Biomedical Sci 5: 139. doi:10.4172/2155- 9538.1000139

Page 3 of 4

\begin{tabular}{|l|c|c|c|c|c|c|}
\hline \multirow{3}{*}{ TB Types } & \multicolumn{7}{|c|}{ Treatment Outcome } \\
\cline { 2 - 7 } & $\begin{array}{c}\text { Cured } \\
\text { N (\%) }\end{array}$ & $\begin{array}{c}\text { Treatment C } \\
\text { N (\%) }\end{array}$ & $\begin{array}{l}\text { Default } \\
\text { N (\%) }\end{array}$ & $\begin{array}{c}\text { Death } \\
\text { N (\%) }\end{array}$ & $\begin{array}{c}\text { Transfer out } \\
\text { N (\%) }\end{array}$ & $\begin{array}{c}\text { TS } \\
\text { N (\%) }\end{array}$ \\
\hline SPPTB & $16(22.9)$ & $7(10.0)$ & $1(1.4)$ & $4(5.7)$ & $42(60.2)$ & $23(32.9)$ \\
\hline SNPTB & - & $69(26.5)$ & $2(0.8)$ & $17(6.5)$ & $173(66.3)$ & $69(26.5)$ \\
\hline EXPTB & - & $23(34.8)$ & $2(3.0)$ & $1(1.5)$ & $40(60.6)$ & $23(34.8)$ \\
\hline Total & 16 & 99 & 5 & 22 & 225 & 397 \\
\hline
\end{tabular}

SPPTB=Smear-Positive Pulmonary TB. SNPTB=Smear-Negative Pulmonary TB. EXPTB=Extrapulmonary TB. TS=Treatment Success

Table 3: Treatment outcomes of HIV infected TB patients ( $N=397)$ in Mizan Aman General Hospital, 2010-2013.

public health problem, especially in resource constraint settings. In this study, the prevalence of HIV co-infection among TB patients was $18.5 \%$; nearly similar to the studies conducted in Brazil (19\%) [8] and in Nigeria (20\%) [14]. However, this finding is lower than that of study conducted in northwest Ethiopia (25\%) [15], and higher than the northwest Ethiopia study (11.4\%) [16] and that of 2013 WHO report for Ethiopia (10\%). Since the study area is gold mining area this increases non stable sexually active people, thus study area signifies the urgent need urgent effective health intervention.

The higher prevalence of co-infection among males in the sexually active age groups in this study, is in agreement with the study conducted in northwest Ethiopia. This can be usually due to exposure of these groups to sexual activities and probably due to the migration of adult male population to the study area for gold mining. Moreover, the greater proportion of SNPTB cases observed among HIV infected TB patients in this study is supported by other study findings [17]. This might be associated with variation in the concentration of acid fast bacilli in the sputum and the rate of caseation necrosis.

The success rate of all cases of TB (29.0\%) in this study were unsatisfactory when compared to the studies conducted in north India that reported a TSR of 77\% [18]. TSR of $66.6 \%$ in HIV infected SPPTB patients was also reported in Nigeria study [19], which is higher than TSR reported in our study (32.9\%). A greater success was reported in one study in Brazil, which observed a $55.8 \%$ cure rate among TB/ HIV co-infected patients. The low treatment success rate observed in this study might be attributed to the high transferred out rate (56.8\%), were treatment outcome of patients who were transferred out were unknown.

Unfavorable treatment outcomes in this study, including default $(1.3 \%)$ and death $(5.5 \%)$ were lower than the findings in other studies. The overall default rate in the current study was lower than that of $8.1 \%$ and $9 \%$ observed among HIV infected TB patients in north India and in Nigeria, respectively. High rate of default is a major problem in the management of patients in the programme and is associated with adverse drug reactions, social stigma and lack of awareness of the disease.

The death rate while on treatment (5.5\%) in this study was lower than death rate reported from India (13.2\%) and Nigeria (16\%) studies. Furthermore, the death rate of SPPTB HIV infected patients in this study was also lower than the study conducted in Brazil. These differences might be due to the high prevalence of transfer out in our study area, which needs additional investigation. In countries with high HIV prevalence, TB deaths are crucial for monitoring programme performance, but are limited by incomplete coverage of all incident $\mathrm{TB}$ cases, inaccurate routine programme reporting of deaths, and the unknown contribution of deaths from TB and HIV alone [20,21].

\section{Conclusion}

A high prevalence of HIV co-infection (18.5\%) was observed among tuberculosis patients treated at Mizan Aman General Hospital in southwest Ethiopia. The treatment success rate of HIV co-infected TB patients was unsatisfactorily low (29\%). A high proportion of patients died $(5.5 \%)$, which is a serious public health concern that needs to be addressed urgently. Thus, to improve treatment outcome of tuberculosis patients TB/HIV integration as well as improved counseling during the intensive and continuation phases of treatment and health education to reduce treatment interruption is required in our setting.

\section{Authors' Contributions}

TF and TG was the primary researcher, conceived the study, designed, participated in data collection, conducted data analysis and drafted the manuscript for publication. HG and YD assisted in data collection, interpretation of the results and preparation of first draft of manuscript. All authors read and approved the final manuscript.

\section{Acknowledgement}

Authors would like to thank all the staff members of TB clinic, Mizan Aman General Hospital for the proper documentation of patient's information and treatment outcome and for their genuine assistance during data collection.

\section{References}

1. Bartlett JG (2007) Tuberculosis and HIV Infection: Partners in Human Tragedy. $\mathrm{J}$ Infect Dis 196: S124-125

2. Global tuberculosis report (2013) World Health Organization, Geneva, Switzerland

3. Corbett EL, Watt CJ, Walker N, Maher D, Williams BG, et al. (2003) The growing burden of tuberculosis: global trends and interactions with the HIV epidemic. Arch Intern Med 163: 1009-1021.

4. Harries A, Maher D, Graham S (2004) TB/HIV: a clinical manual. World Health Organization, Geneva.

5. Pawlowski A, Jansson M, Skold M, Rottenberg ME, Kallenius G (2012) Tuberculosis and HIV Co-Infection. PLoS Pathog 8: e1002464.

6. Odhiambo JA, Borgdorff MW, Kiambih FM (1999) Tuberculosis and the HIV epidemic: increasing annual risk of infection in Kenya, 1986-1996. Am J Public Heal 89: 1078-1082.

7. Dolin PJ, Raviglione MC, Kochi A (1994) Global tuberculosis incidence and mortality during 1990-2000. Bull World Heal Organ 72:213-220.

8. do Prado TN, Miranda AE, de Souza FM, dos Santos Dias E, Fernandes Sousa LK, et al. (2014) Factors associated with tuberculosis by HIV status in the Brazilian national surveillance system: a cross sectional study. BMC Infect Dis14: 415.

9. Tweya H, Feldacker C, Phiri S, Ben-Smith A, Fenner L, et al. (2013) Comparison of Treatment Outcomes of New Smear-Positive Pulmonary Tuberculosis Patients by HIV and Antiretroviral Status in a TB/HIV Clinic, Malawi. PLoS ONE 8: e56248.

10. Elliott AM, Halwiindi B, Hayes RJ, Luo N, Mwinga AG, et al. (1995) The impact of human immunodeficiency virus on mortality of patients treated fo tuberculosis in a cohort study in Zambia. Trans R Soc Trop Med Hyg 89: 78-82.

11. Korenromp EL, Scano F, Williams BG, Dye C, Nunn P (2003) Effects of human immunodeficiency virus infection on recurrence of tuberculosis after rifampinbased treatment: an analytical review. Clin Infect Dis 37: 101

12. Ministry of Health of Ethiopia (MOH) (2008) Tuberculosis, Leprosy and TB/HIV Prevention and Control Programme Manual. Addis Ababa.

13. Ministry of Health of Ethiopia (MOH) (2009) Tuberculosis, Leprosy and TB/HIV Prevention and Control Programme Manual. Addis Ababa.

14. Babatunde O, Elegbede O, Ayodele M, Fadare J, Isinjaye A, et al. (2013) Factors Affecting Treatment Outcomes of Tuberculosis in a Tertiary Health Center in Southwestern Nigeria. Int Rev Soc Sci Humanit 4: 209-218. 
Citation: Fiseha T, Gebru T, Gutema H, Debela Y (2015) Tuberculosis Treatment Outcome among HIV Co-infected Patients at Mizan-Aman General Hospital, Southwest Ethiopia: A Retrospective Study. J Bioengineer \& Biomedical Sci 5: 139. doi:10.4172/2155- 9538.1000139

15. Biadglegne F, Anagaw B, Debebe T, Anagaw B, Tesfaye W, et al. (2013) A retrospective study on the outcomes of tuberculosis treatment in Felege Hiwot Referral Hospital, Northwest Ethiopia. Int J Med Med Sci 5: 85-91.

16. Tadesse S, Tadesse T (2013) HIV co-infection among tuberculosis patients in Dabat, northwest Ethiopia. J Infect Dis Immun 5: 29-32.

17. Wondimeneh Y, Muluye D, Belyhun S (2012) Prevalence of pulmonary tuberculosis and immunological profile of HIV co-infected patients in Northwest Ethiopia. BMC Res Notes 5: 331.

18. Sharma SK, Soneja M, Prasad KT, Ranjan S (2014) Clinical profile \& predictors of poor outcome of adult HIV-tuberculosis patients in a tertiary care centre in north India. Indian J Med Res 139: 154-160.

19. Dauda MM (2010) Evaluation of the efficacy of directly observed treatment short course (DOTS) in patients with tuberculosis and HIV Co-infection in Kano. Nigeria. Rev Infect 1: 218-223.

20. Maher D, Watt CJ, Williams BG, Raviglione M, Dye C (2005) Tuberculosis deaths in countries with high HIV prevalence: what is their use as an indicator in tuberculosis programme monitoring and epidemiological surveillance? Unresolved Issues. Int J Tuberc Lung Dis 9: 123-127.

21. Havlir DV, Getahun H, Sanne I (2008) Opportunities and challenges for HIV care in overlapping HIV and TB epidemics. JAMA 300: 423-430. 